\title{
Balanced Fiber Bundles and GKM Theory
}

\section{Victor Guillemin ${ }^{1}$, Silvia Sabatini ${ }^{2}$, and Catalin Zara ${ }^{3}$}

${ }^{1}$ Department of Mathematics, MIT, Cambridge, MA 02139, USA, ${ }^{2}$ Department of Mathematics, EPFL, Lausanne, Switzerland, and ${ }^{3}$ Department of Mathematics, University of Massachusetts, Boston, MA 02125, USA

Correspondence to be sent to:vwg@math.mit.edu

Let $T$ be a torus and $B$ a compact $T$-manifold. Goresky et al. show in [3] that if $B$ is (what was subsequently called) a GKM manifold, then there exists a simple combinatorial description of the equivariant cohomology ring $H_{T}^{*}(B)$ as a subring of $H_{T}^{*}\left(B^{T}\right)$. In this paper, we prove an analog of this result for $T$-equivariant fiber bundles: we show that if $M$ is a $T$-manifold and $\pi: M \rightarrow B$ a fiber bundle for which $\pi$ intertwines the two $T$-actions, there is a simple combinatorial description of $H_{T}^{*}(M)$ as a subring of $H_{T}^{*}\left(\pi^{-1}\left(B^{T}\right)\right)$. Using this result, we obtain fiber bundle analogs of results of Guillemin et al. [4] on GKM theory for homogeneous spaces.

\section{Introduction}

Let $T=\left(S^{1}\right)^{n}$ be an $n$-dimensional torus and $M$ a compact, connected $T$-manifold. We recall that the equivariant cohomology $H_{T}^{*}(M)=H_{T}^{*}(M ; \mathbb{R})$ of $M$ is defined as the usual cohomology of the quotient $(M \times E) / T$, where $E$ is the total space of the classifying bundle of the group $T$. Let

$$
\pi: M \rightarrow B
$$

be a $T$-equivariant fiber bundle. We will assume that the base $B$ is simply connected and that the typical fiber is connected.

Received December 7, 2011; Revised May 1, 2012; Accepted June 8, 2012

(C) The Author(s) 2012. Published by Oxford University Press. All rights reserved. For permissions, please e-mail: journals.permissions@oup.com. 
Then one obtains a fiber bundle

$$
(M \times E) / T \rightarrow(B \times E) / T
$$

and a Serre-Leray spectral sequence relating the equivariant cohomology groups of $M$ and $B$; the $E_{2}$-term of this spectral sequence is the product

$$
H^{*}(F) \otimes H^{*}((B \times E) / T)
$$

where $F$ is the fiber of the bundle (1.2) and hence of the bundle (1.1). Thus, if the spectral sequence collapses at this stage, one obtains an isomorphism of additive cohomology

$$
H_{T}^{*}(M) \simeq H^{*}(F) \otimes H_{T}^{*}(B)
$$

However, this isomorphism does not say much about how the ring structure of $H_{T}^{*}(B)$ and $H_{T}^{*}(M)$ are related. One of the main goals of this paper is to address that question. We begin by recalling that one approach for computing the equivariant cohomology ring of a $T$-manifold $M$ is by Kirwan localization. Suppose that $H_{T}^{*}(M)$ is free as a module over $H_{T}^{*}(p t) \simeq \mathbb{S}\left(\mathfrak{t}^{*}\right)$, the symmetric algebra of $\mathfrak{t}^{*}$. Then the restriction map

$$
i^{*}: H_{T}^{*}(M) \rightarrow H_{T}^{*}\left(M^{T}\right)
$$

is injective and hence computing $H_{T}^{*}(M)$ reduces to computing the image of $H_{T}^{*}(M)$ in $H_{T}^{*}\left(M^{T}\right)$. If $M^{T}$ is finite, then

$$
H_{T}^{*}\left(M^{T}\right) \simeq \bigoplus_{p \in M^{T}} \mathbb{S}\left(\mathfrak{t}^{*}\right)
$$

Determining where $H_{T}^{*}(M)$ sits inside this sum is a challenging combinatorial problem. However, one class of spaces for which this problem has a simple and elegant solution is the one introduced by Goresky-Kottwitz-MacPherson in their seminal paper [3]. These are now known as GKM spaces.

Definition 1.1. A compact $T$-manifold $M$ is a GKM manifold if:

(a) $M^{T}$ is finite. 
(b) $M$ is equivariantly formal, that is,

$$
H_{T}^{*}(M) \simeq H^{*}(M) \otimes \mathbb{S}\left(\mathfrak{t}^{*}\right)
$$

as $\mathbb{S}\left(\mathfrak{t}^{*}\right)$-modules.

(c) For every codimension one subtorus $T^{\prime} \subset T$, the connected components of $M^{T^{\prime}}$ are either points or two-spheres.

If $S$ is one of the edge two-spheres, then $S^{T}$ consists of exactly two $T$-fixed points, $p$ and $q$ (the "North" and "South" poles of $S$ ). To each GKM space $M$ we attach a graph $\Gamma=\Gamma_{M}$ with set of vertices $V_{\Gamma}=M^{T}$, and edges corresponding to these two-spheres. If $M$ has an invariant almost complex or symplectic structure, then the isotropy representations on tangent spaces at fixed points are complex representations and their weights are well-defined. In particular, condition (c) is equivalent to:

(c') For every fixed point $p \in M^{T}$, the weights of the isotropy representation of $T$ on $T_{p} M$ are pairwise linearly independent.

These data determine a map

$$
\alpha: E_{\Gamma} \rightarrow \mathbb{Z}_{T}^{*}
$$

of oriented edges of $\Gamma$ into the weight lattice of $T$. This map assigns to the edge (two-sphere) $S$, joining $p$ to $q$ and oriented from $p$ to $q$, the weight of the isotropy representation of $T$ on the tangent space to $S$ at $p$. The map $\alpha$ is called the axial function of the graph $\Gamma$. We use it to define a subring $H_{\alpha}^{*}(\Gamma)$ of $H_{T}^{*}\left(M^{T}\right)$ as follows. Each element of $H_{T}^{*}\left(M^{T}\right)$ can be regarded as a function that assigns to each $p \in M^{T}$ an element $c(p)$ of $H_{T}^{*}(p t)=\mathbb{S}\left(\mathfrak{t}^{*}\right)$, hence $H_{T}^{*}\left(M^{T}\right)$ is identified with the $\operatorname{ring} \operatorname{Maps}\left(V_{\Gamma}, \mathbb{S}\left(\mathfrak{t}^{*}\right)\right)$.

Definition 1.2. The cohomology ring of $(\Gamma, \alpha)$, denoted by $H_{\alpha}^{*}(\Gamma)$, is the subring of $\operatorname{Maps}\left(V_{\Gamma}, \mathbb{S}\left(\mathfrak{t}^{*}\right)\right)$ consisting of the maps $c$ that have the property that for each edge $e$ of $\Gamma$, with vertices $p$ and $q$ as endpoints, $c(p)$ and $c(q)$ have the same image in $\mathbb{S}\left(\mathfrak{t}^{*}\right) /\left\langle\alpha_{e}\right\rangle$.

Note that without the invariant almost complex or symplectic structure, the isotropy representations are only real representations and the weights are defined only up to sign; however, that does not change the construction of $H_{\alpha}^{*}(\Gamma)$. 
For GKM spaces, a direct consequence of a theorem of Chang and Skjelbred [2, Lemma 2.3] is that $H_{\alpha}^{*}(\Gamma)$ is the image of $i^{*}$ (see [3, Theorem 1.2.2]), and therefore there is an isomorphism of rings

$$
H_{T}^{*}(M) \simeq H_{\alpha}^{*}(\Gamma)
$$

One of our main results is a generalization of (1.5) for $T$-equivariant fiber bundles

$$
\pi: M \rightarrow B
$$

for which the total space $M$ is equivariantly formal and the base $B$ is a GKM space. By the Kirwan Theorem, the composite map

$$
H_{T}^{*}(M) \rightarrow H_{T}^{*}\left(\pi^{-1}\left(B^{T}\right)\right) \rightarrow H_{T}^{*}\left(M^{T}\right)
$$

is injective. Hence one has an injective homomorphism of rings

$$
H_{T}^{*}(M) \rightarrow \bigoplus_{p \in B^{T}} H_{T}^{*}\left(\pi^{-1}(p)\right)
$$

and so to determine the ring structure of $H_{T}^{*}(M)$ it suffices to determine the image of this mapping. This we will do by a GKM type recipe similar to (1.5).

Let $\left(\Gamma=\Gamma_{B}, \alpha\right)$ be the GKM graph associated to $B$, and for $p \in B^{T}$ (i.e., a vertex of $\Gamma)$ let $F_{p}=\pi^{-1}(p)$. If $e$ is an edge of $\Gamma$ joining the vertices $p$ and $q$, and $T_{e}$ is the subtorus of $T$ with Lie algebra $\operatorname{ker} \alpha_{e}$, then $F_{p}$ and $F_{q}$ are isomorphic as $T_{e}$-spaces and hence

$$
H_{T}^{*}\left(F_{p}\right) /\left\langle\alpha_{e}\right\rangle=H_{T}^{*}\left(F_{q}\right) /\left\langle\alpha_{e}\right\rangle
$$

We denote the ring (1.8) by $\mathcal{R}_{e}$ and we will prove the following generalization of (1.5).

Theorem 1.3. A function

$$
c: V_{\Gamma} \rightarrow \bigoplus_{p \in B^{T}} H_{T}^{*}\left(F_{p}\right), c(p) \in H_{T}^{*}\left(F_{p}\right)
$$

is in the image of (1.7) if and only if for every edge $e=(p, q)$ of $\Gamma$, the images of $c(p)$ and $c(q)$ in $\mathcal{R}_{e}$ coincide.

One of our main applications of this result will be a fiber bundle version of the main result in [4]. In [4], it is shown that if $G$ is a compact semisimple Lie group, $T$ a Cartan subgroup, and $K$ a closed subgroup of $G$, then the following conditions 
are equivalent:

(1) the action of $T$ on $G / K$ is GKM;

(2) the Euler characteristic of $G / K$ is nonzero;

(3) $K$ is of maximal rank, that is, $T \subset K$.

Moreover, for homogeneous spaces of the form $G / K$ one has a description, due to Borel, of the equivariant cohomology ring of $G / K$ as a tensor product

$$
H_{T}^{*}(G / K)=\mathbb{S}\left(\mathfrak{t}^{*}\right)^{W_{K}} \otimes_{\mathbb{S}\left(\mathfrak{t}^{*}\right)^{W_{G}}} \mathbb{S}\left(\mathfrak{t}^{*}\right)
$$

and in [4] it is shown how to reconcile (1.9) with the description (1.5).

Suppose now that $M \rightarrow B$ is a $T$-equivariant fiber bundle with $M$ equivariantly formal and $B$ a GKM space. Let $p \in B^{T}$ be a fixed point for the $T$-action on $B$ and $\pi_{1}(\Gamma, p)$ the group of paths $\left(e_{1}, e_{2}, \ldots, e_{k}\right)$ of oriented edges on $\Gamma$ starting and ending at $p$. Modulo some hypotheses which we will spell out more carefully in Section 4 , the group $\pi_{1}(\Gamma, p)$ induces an action of a group $W_{p}$ (which we will call the Weyl group of $p$ ) both on $H_{T}^{*}\left(F_{p}\right)$ and on $\mathbb{S}\left(\mathfrak{t}^{*}\right)$. Fiber bundles satisfying those conditions will be called balanced. We will then show that there is a canonical embedding of $H_{T}^{*}\left(F_{p}\right)^{W_{p}}$ into $H_{T}^{*}(M)$ and its image generates $H_{T}^{*}(M)$ as a module over $H_{T}^{*}(B)$. One of the main results of this paper (see Theorem 4.2) is the following: if $\Gamma_{B}$ is the GKM graph of $B$, then there is a canonical isomorphism of rings

$$
H_{T}^{*}(M) \simeq H_{T}^{*}\left(F_{p}\right)^{W_{p}} \otimes_{\mathbb{S}\left(\mathbf{t}^{*}\right)^{W_{p}}} H_{\alpha}\left(\Gamma_{B}\right) .
$$

We apply this result to fiber bundles of the type $G / K_{1} \rightarrow G / K$, with $K_{1} \subset K$, and obtain a description of the cohomology ring of $G / K_{1}$ that will be of Borel type on the fibers and of GKM type on the base. This result will enable one to interpolate between two (in principle) very different descriptions of the ring $H_{T}^{*}(G / K)$, as we have already shown in special cases in [5].

A few words about the organization of this paper. In Section 2, we generalize the Chang-Skjelbred theorem to equivariant fiber bundles, and in Section 3 we use this result to prove Theorem 1.3. In Section 4, we construct the group $W_{p}$ and prove the isomorphism (1.10). In Section 5, we describe in more detail the results of Guillemin et al. [4] alluded to above and show that the fiber bundles $G / K_{1} \rightarrow G / K$ satisfy the conditions of Theorem 4.2, and in Section 6 we describe some connections between the results of this paper and results of Guillemin et al. [5], where we work out the implications of this theory in much greater detail for the classical flag varieties of type $A_{n}, B_{n}, C_{n}$, and $D_{n}$.

The results of Section 6 are also related to those of [9], in which the authors use symplectic techniques to compute the equivariant cohomology ring of a compact 
symplectic manifold with a Hamiltonian torus action that fibers equivariantly over another compact symplectic manifold with a Hamiltonian action of the same torus. In particular, the details of classical flag varieties are also analyzed.

The results of this paper are closely related to the results of [6? ], the topic of which is $K$-theoretic aspects of GKM theory. In particular, in [6], we have shown how the graph theoretical analogs of the results of this paper can be extended to the $K$-theoretic setting, and in some work-in-progress we have also been able to show that there is $K$-theoretic version of the Chang-Skjelbred theorem of Section 2 and that it gives one an effective way of computing the $K$-groups of balanced fiber bundles.

To conclude we would like to thank Sue Tolman, Allen Knutson, and Tara Holm for helpful comments and, above all, thank the referee for a number of expositional suggestions that have considerably improved the readability of Sections 4 and 6 .

\section{The Chang-Skjelbred Theorem for Fiber Bundles}

Before proving the main theorem of this section, we recall the Chang-Skjelbred theorem [2, Lemma 2.3].

Let $M$ be a compact manifold with a smooth action of a compact torus $T$, and assume that $M$ is equivariantly formal. Let $K \subset T$ be an isotropy group of $M$, that is, there exists $x \in M$ such that $K=\{g \in T \mid g \cdot x=x\}$. From the compactness of $M$ it follows that the family of groups which occur as isotropy groups of $M$ is finite. Let $K_{1}, \ldots, K_{N}$ be the family of codimension one isotropy groups of $M$, and $M^{K_{j}}$ the subset of points of $M$ fixed by $K_{j}$, for every $j=1, \ldots, N$. From the commutativity of the diagram given by inclusions of $T$-invariant spaces

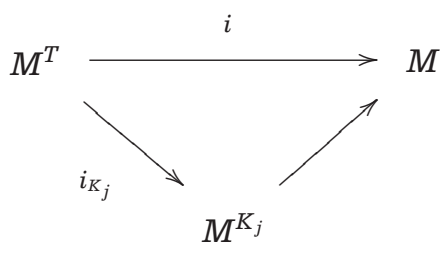

we obtain the following commutative diagram in equivariant cohomology:

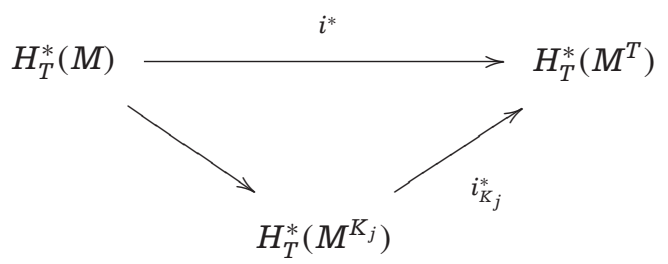


which implies that

$$
i^{*}\left(H_{T}^{*}(M)\right) \subseteq \bigcap_{j=1}^{N} i_{K_{j}}^{*}\left(H_{T}^{*}\left(M^{K_{j}}\right)\right) .
$$

Lemma 2.3 in [2] asserts that the opposite inclusion holds as well.

Theorem 2.1 (Chang-Skjelbred). Let $M$ be a compact $T$-manifold, and assume that $M$ is equivariantly formal. Let $K_{1} \ldots, K_{N}$ be the subtori of codimension one which occur as isotropy groups of points of $M$. Then

$$
i^{*}\left(H_{T}^{*}(M)\right)=\bigcap_{j=1}^{N} i_{K_{j}}^{*}\left(H_{T}^{*}\left(M^{K_{j}}\right)\right) .
$$

Before proving the main theorem of this section we recall some standard facts about T-manifolds. (For a detailed exposition we refer to [7, Sections 11.3-4]; see also [1].) Let $A$ be a finitely generated $\mathbb{S}\left(\mathfrak{t}^{*}\right)$-module. The annihilator ideal $I_{A}$ of $A$ is

$$
I_{A}=\left\{f \in \mathbb{S}\left(\mathfrak{t}^{*}\right), f A=0\right\}
$$

and the support of $A$ is the algebraic variety in $\mathfrak{t} \otimes \mathbb{C}$ associated with this ideal, that is,

$$
\operatorname{supp} A=\left\{x \in \mathfrak{t} \otimes \mathbb{C}, f(x)=0 \text { for all } f \in I_{A}\right\}
$$

Theorem 2.2 ([7, Theorem 11.4.1]). Let $M$ be a compact $T$-manifold and $X$ a closed $T$-invariant submanifold. Then the supports of the modules $H_{T}^{*}(M \backslash X)$ and $H_{T}^{*}(M \backslash X)_{C}$ are contained in the set

$$
\bigcup_{K} \mathfrak{k} \otimes \mathbb{C}
$$

the union being over the subgroups $K$ which occur as isotropy groups of points of $M \backslash X$.

Here, $H_{T}^{*}(\cdot)_{C}$ denotes the equivariant cohomology with compact supports. Moreover, the following sequence:

$$
H_{T}^{k}\left(M \backslash X^{T}\right)_{c} \longrightarrow H_{T}^{k}(M) \longrightarrow H_{T}^{k}\left(X^{T}\right) \longrightarrow H_{T}^{k+1}\left(M \backslash X^{T}\right)_{c}
$$


is exact.

Theorem 2.3 ([7, Theorem 11.4.2]). Let $M$ and $X$ be as in Theorem 2.2, and $i: X \rightarrow M$ the inclusion. The kernel and cokerel of the map $i^{*}: H_{T}^{*}(M) \rightarrow H_{T}^{*}(X)$ are supported in the set (2.2).

Let $\pi: M \rightarrow B$ be a $T$-equivariant fiber bundle with $M$ equivariantly formal and $B$ a GKM space. Let $K_{j}, j=1, \ldots, N$ be the codimension one isotropy groups of $B$ and let $\mathfrak{k}_{j}$ be the Lie algebra of $K_{j}$.

Lemma 2.4. Let $x \in B, K=\{g \in T \mid g \cdot x=x\}$ its isotropy group, and $\mathfrak{k}$ the Lie algebra of $K$. Then

$$
\mathfrak{k}=\bigcap_{r=1}^{m} \mathfrak{k}_{j_{r}}
$$

for some multi-index $1 \leqslant j_{1}<\cdots<j_{m} \leqslant N$.

Proof. Let $Y \subset B^{K} \subset B$ be the connected component through $x$ of the subspace fixed by $K$. The tangent space at $X, T_{X} B$, has a natural $T$ action, and can be written as the direct sum of two $T$-invariant subspaces $T_{X} Y \oplus N_{X}$, where $T_{X} Y$ denotes the tangent space of $Y$ at $X$, and $N$ is the normal bundle with respect to a $T$-invariant metric on $B$. On the other hand,

$$
T_{X} B=\bigoplus_{j=1}^{n} \mathbb{L}_{\alpha_{j}}
$$

for some $\alpha_{1}, \ldots, \alpha_{n} \in \mathfrak{t}^{*}$, where $\mathbb{L}_{\alpha_{j}}$ denotes the tangent space to the $T$-invariant sphere containing $x$, fixed by $\exp \left(\operatorname{ker}\left(\alpha_{j}\right)\right) \subset T$, for every $j=1, \ldots, n$. Thus

$$
T_{X} Y=\bigoplus_{r=1}^{m} \mathbb{L}_{\alpha_{j_{r}}}
$$

for some multi-index $1 \leqslant j_{1}<\cdots<j_{m} \leqslant N$ and

$$
\mathfrak{k}=\bigcap_{r=1}^{m} \operatorname{ker}\left(\alpha_{j_{r}}\right)=\bigcap_{r=1}^{m} \mathfrak{k}_{j_{r}} .
$$

For any subgroup $K$ of $T$ let $X^{K}=\pi^{-1}\left(B^{K}\right)$, where $B^{K} \subset B$ is the set of points in $B$ fixed by $K$. Then $X^{K}$ is a closed $T$-invariant submanifold of $M$. In particular, 
$X^{T}=\pi^{-1}\left(B^{T}\right)$, the inverse image of the $T$-fixed points of $B$. From Lemma 2.4 and Theorem 2.2 one obtains the following.

Theorem 2.5. The $\mathbb{S}\left(\mathfrak{t}^{*}\right)$-module $H_{T}^{*}\left(M \backslash X^{T}\right)$ is supported on the set

$$
\bigcup_{i=1}^{N} \mathfrak{k}_{i} \otimes \mathbb{C} .
$$

Since $H_{T}^{*}(M)$ is a free $\mathbb{S}\left(t^{*}\right)$-module, by the exactness of (2.3) and Theorem 2.5 we have the following theorem.

Theorem 2.6. Let $i: X^{T} \rightarrow M$ be the inclusion, and $i^{*}: H_{T}(M) \rightarrow H_{T}\left(X^{T}\right)$ the induced map in equivariant cohomology. Then $i^{*}$ is injective and $\operatorname{coker}\left(i^{*}\right)$ is supported on $\bigcup_{i=1}^{N} \mathfrak{k}_{i} \otimes \mathbb{C}$.

As a consequence, we obtain the following corollary.

Corollary 2.7. If $e \in H_{T}^{*}\left(X^{T}\right)$, then there exist nonzero weights $\alpha_{1}, \ldots, \alpha_{r}$ such that $\alpha_{j}=0$ on some $\mathfrak{k}_{j}$ and

$$
\alpha_{1} \cdots \alpha_{r} e \in i^{*}\left(H_{T}^{*}(M)\right)
$$

The next theorem is a fiber bundle version of the Chang-Skjelbred theorem.

Theorem 2.8. Let $i: X^{T} \rightarrow M$ be the inclusion, and $i^{*}: H_{T}(M) \rightarrow H_{T}\left(X^{T}\right)$ the induced map in equivariant cohomology. Then the image of $i^{*}$ is the ring

$$
\bigcap_{j=1}^{N} i_{K_{j}}^{*} H_{T}^{*}\left(X^{K_{j}}\right),
$$

where $i_{K_{j}}$ denotes the inclusion of $X^{T}$ into $X^{K_{j}}$.

Proof. Via the inclusion $i^{*}$ we can view $H_{T}^{*}(M)$ as a submodule of $H_{T}^{*}\left(X^{T}\right)$. Let $e_{1}, \ldots, e_{m}$ be a basis of $H_{T}^{*}(M)$ as a free module over $\mathbb{S}\left(\mathfrak{t}^{*}\right)$. Then by Corollary 2.7 for any $e \in H_{T}^{*}\left(X^{T}\right)$ 
we have

$$
\alpha_{1} \cdots \alpha_{r} e=\sum f_{j} e_{j}, \quad f_{j} \in \mathbb{S}\left(\mathfrak{t}^{*}\right)
$$

Then $e=\sum \frac{f_{j}}{p} e_{j}$, where $p=\alpha_{1} \cdots \alpha_{r}$. If $f_{j}$ and $p$ have a common factor we can eliminate it and write $e$ uniquely as

$$
e=\sum \frac{g_{j}}{p_{j}} e_{j}
$$

with $g_{j} \in \mathbb{S}\left(\mathrm{t}^{*}\right), p_{j}$ a product of a subset of the weights $\alpha_{1}, \ldots, \alpha_{r}$ and $p_{j}$ and $g_{j}$ relatively prime.

Suppose that $K$ is an isotropy subgroup of $B$ of codimension one and $e$ is in the image of $H_{T}^{*}\left(X^{K}\right)$. By Theorem 2.3 the cokernel of the map $H_{T}^{*}(M) \rightarrow H_{T}^{*}\left(X^{K}\right)$ is supported on the subset $\cup \mathfrak{k}_{j} \otimes \mathbb{C}, \mathfrak{k}_{j} \neq \mathfrak{k}$ of (2.4), and hence there exists weights $\beta_{1}, \ldots, \beta_{r}, \beta_{j}$ vanishing on some $\mathfrak{k}_{j}$ but not on $\mathfrak{k}$, such that

$$
\beta_{i} \cdots \beta_{r} e=\sum f_{j} e_{j}
$$

Thus the $p_{j}$ in (2.7), which is a product of a subset of the weights $\alpha_{1}, \ldots, \alpha_{r}$, is a product of a subset of weights none of which vanish on $\mathfrak{k}$. Repeating this argument for all the codimension one isotropy groups of $B$ we conclude that the weights in this subset cannot vanish on any of these $\mathfrak{k}^{\prime} \mathbf{s}$, and hence is the empty set, that is, $p_{j}=1$. Then if $e$ is in the intersection (2.6), $e$ is in $H_{T}^{*}(M)$.

Remark 2.9. Observe that Theorem 2.1 in the case in which $M$ is GKM is a special case of Theorem 2.8, obtained for $M=B$ and $\pi$ the identity.

\section{Fiber Bundles over GKM Spaces}

We can now prove Theorem 1.3, the fiber bundle version of the GKM result.

We start with the case $B=\mathbb{C} P^{1}$. Let $\pi: M \rightarrow B=\mathbb{C} P^{1}$ be a $T$-equivariant fiber bundle with $M$ equivariantly formal. If $T$ acts on $\mathbb{C} P^{1}$ with weight $\alpha_{e}$, then the codimension one subtorus $T_{e}=\exp \left(\operatorname{ker} \alpha_{e}\right) \subset T$ fixes the points of $B$, and the action of the quotient group $T / T_{e}$ is effective. The fixed point set $B^{T}$ consists of two points, $p$ and $q$ and its inverse image under $\pi, X^{T}=\pi^{-1}\left(B^{T}\right)$, consists of the two fibers $F_{p}=\pi^{-1}(p)$ and $F_{q}=\pi^{-1}(q)$. If $i: X^{T} \rightarrow M$ is the inclusion, then the map $i^{*}: H_{T}^{*}(M) \rightarrow H_{T}^{*}\left(X^{T}\right)$ is injective 
(see (1.7)). Recall that $F_{p}$ and $F_{q}$ are isomorphic as $T_{e}$-spaces and that (see (1.8))

$$
H_{T}^{*}\left(F_{p}\right) /\left\langle\alpha_{e}\right\rangle=H_{T_{e}}^{*}\left(F_{p}\right)=H_{T_{e}}^{*}\left(F_{q}\right)=H_{T}^{*}\left(F_{q}\right) /\left\langle\alpha_{e}\right\rangle=\mathcal{R}_{e}
$$

Let $r_{p}$ and $r_{q}$ be the projections of $H_{T}^{*}\left(F_{p}\right)$ and $H_{T}^{*}\left(F_{q}\right)$, respectively, onto $\mathcal{R}_{e}$.

Theorem 3.1. The image of the map

$$
H_{T}^{*}(M) \rightarrow H_{T}^{*}\left(X^{T}\right)
$$

is the set of pairs $\left(f_{p}, f_{q}\right) \in H_{T}^{*}\left(F_{p}\right) \oplus H_{T}^{*}\left(F_{q}\right)$ satisfying $r_{p}\left(f_{p}\right)=r_{q}\left(f_{q}\right)$.

Proof. Let $S^{1}$ be a circle complement of $T_{e}$ in $T$, that is, $T=T_{e} \times S^{1}$. Then $S^{1}$ acts freely on $\mathbb{C} P^{1} \backslash\{p, q\}$ and the quotient by $S^{1}$ of this action is the interval $(0,1)$, so one has an isomorphism of $T_{e}$ spaces

$$
\left(M \backslash X^{T}\right) / S^{1}=F \times(0,1),
$$

where, as $T_{e}$-spaces, $F=F_{p}=F_{q}$.

Consider now the long exact sequence (2.3). Since $i^{*}$ is injective this becomes a short exact sequence

$$
0 \rightarrow H_{T}^{k}(M) \rightarrow H_{T}^{k}\left(X^{T}\right) \rightarrow H_{T}^{k+1}\left(M \backslash X^{T}\right)_{C} \rightarrow 0 .
$$

Since $S^{1}$ acts freely on $M \backslash X^{T}$, we have

$$
H_{T}^{k+1}\left(M \backslash X^{T}\right)_{c}=H_{T_{e}}^{k+1}\left(\left(M \backslash X^{T}\right) / S^{1}\right)_{C}
$$

and by fiber integration one obtains from (3.1)

$$
H_{T_{e}}^{k+1}\left(\left(M \backslash X^{T}\right) / S^{1}\right)_{C}=H_{T_{e}}^{k}(F) .
$$

Then the sequence (3.2) becomes

$$
0 \rightarrow H_{T}^{k}(M) \rightarrow H_{T}^{k}\left(F_{p}\right) \oplus H_{T}^{k}\left(F_{q}\right) \rightarrow H_{T_{e}}^{k}(F) \rightarrow 0
$$


where the third arrow is the map

$$
H_{T}^{*}\left(X^{T}\right)=H_{T}^{*}\left(F_{p}\right) \oplus H_{T}^{*}\left(F_{q}\right) \rightarrow H_{T_{e}}^{*}(F)
$$

sending $f_{p} \oplus f_{q}$ to $-r_{p}\left(f_{p}\right)+r_{q}\left(f_{q}\right)$. (The $-r_{p}$ in the first term is due to the fact that the fiber integral

$$
H_{C}^{k+1}(F \times(0,1)) \rightarrow H^{k}(F)
$$

depends on the orientation of $(0,1)$ : the standard orientation for $F_{q} \times(0,1) \rightarrow F_{q}$ and the reverse orientation for $F_{p} \times(0,1) \rightarrow F_{p}$.

Theorem 1.3 follows from Theorem 2.8 by applying Theorem 3.1 to all edges of the GKM graph of $B$.

\section{Holonomy for Balanced Bundles}

The goal of this section is to prove one of the main results of this paper, namely the fiber bundle analog of (1.9) we mentioned in the introduction (see (1.10)).

We begin by recalling the Cartan model for equivariant cohomology, considering the special case of torus actions. Let $M$ be a smooth manifold and $\rho: T \times M \rightarrow M$ a smooth action of a torus $T$ on $M$. Let $\mathfrak{t}$ be the Lie algebra of $T$, and for $\xi \in \mathfrak{t}$, let $\xi_{M}$ be the corresponding vector field on $M$, given at $x \in M$ by

$$
\xi_{M}(x)=\left.\frac{\mathrm{d}}{\mathrm{d} t}\right|_{t=0} \rho(\exp (t \xi), x)
$$

The space of cochains

$$
\Omega_{T}(M)=\Omega(M)^{T} \otimes \mathbb{S}\left(\mathfrak{t}^{*}\right)
$$

can be regarded as the space of $T$-invariant polynomial maps

$$
p: \mathfrak{t} \rightarrow \Omega(M),
$$

from $t$ to the space of differential forms $\Omega(M)$. 
The coboundary operator $\mathrm{d}_{T}: \Omega_{T}(M) \rightarrow \Omega_{T}(M)$ is given by

$$
\left(\mathrm{d}_{T} p\right)(\xi)=\mathrm{d}(p(\xi))+\iota\left(\xi_{M}\right) p(\xi),
$$

where $\mathrm{d}$ is the usual differential and $\iota$ is the interior product of a form by a vector field. Then $H_{T}^{*}(M)$ is the cohomology of the complex $\left(\Omega_{T}(M), \mathrm{d}_{T}\right)$.

If $\tau: T \rightarrow T$ is an automorphism of $T$, then we define a second action of $T$ on $M, \rho^{\tau}: T \times M \rightarrow M, \rho^{\tau}(a, x)=\rho(\tau(a), x)$. Let $\left(\Omega_{T}(M)^{\tau}, \mathrm{d}_{T}^{\tau}\right)$ be the complex associated to $\rho^{\tau}$ and $H_{T}^{*}(M)^{\tau}$ the corresponding equivariant cohomology ring. The invariant forms are the same, $\Omega_{T}(M)^{\tau}=\Omega_{T}(M)$, but the coboundary operators are in general different:

$$
\left(\mathrm{d}_{T}^{\tau}(p)\right)(\xi)=\mathrm{d}(p(\xi))+\iota\left(\xi_{M}^{\tau}\right) p(\xi)=\mathrm{d}(p(\xi))+\iota\left(\left(\tau_{*}(\xi)\right)_{M}\right) p(\xi),
$$

where $\tau_{*}: t \rightarrow t$ is the corresponding automorphism of the Lie algebra $t$.

The automorphism $\tau$ induces an isomorphism $\tau^{*}: \Omega_{T}(M) \rightarrow \Omega_{T}(M)$,

$$
\left(\tau^{*}(p)\right)(\xi)=p\left(\tau_{*}(\xi)\right)
$$

and the isomorphism $\tau^{*}$ commutes with the coboundary operators,

$$
\tau^{*} \mathrm{~d}_{T}=\mathrm{d}_{T}^{\tau} \tau^{*}
$$

and therefore it induces an isomorphism $\tau^{*}: H_{T}^{*}(M) \rightarrow H_{T}^{*}(M)^{\tau}$.

Suppose now that $M$ and $N$ are $T$-manifolds and $f: M \rightarrow N$ is a smooth map that intertwines the $T$-action on $M$ with the $\tau$-twisted action on $N$,

$$
f(a \cdot x)=\tau(a) \cdot f(x),
$$

for all $a \in T$ and $x \in M$. The pull-back map $f^{*}: \Omega(N) \rightarrow \Omega(M)$ satisfies

$$
\rho_{M, a}^{*} \circ f^{*}=f^{*} \circ \rho_{N, \tau(a)}^{*},
$$

and extends to a map $f^{*}: \Omega_{T}(N) \rightarrow \Omega_{T}(M)$ by $\left(f^{*} p\right)(\xi)=f^{*}(p(\xi))$, and this map intertwines the $\tau^{*}$ operations for $M$ and $N$,

$$
\tau^{*} f^{*}=f^{*} \tau^{*}
$$


Moreover, $\iota\left(\xi_{M}\right) f^{*}=f^{*} \iota\left(\tau_{*}(\xi)_{N}\right)$ implies that

$$
\mathrm{d}_{T} f^{*}=f^{*} \mathrm{~d}_{T}^{\tau},
$$

where $\mathrm{d}_{T}$ is on $M$ and $\mathrm{d}_{T}^{\tau}$ on $N$.

Thus by (4.3) and (4.5) $\tau^{*} f^{*}$ intertwines the $\mathrm{d}_{T}$ operators on $\Omega_{T}(N)$ and $\Omega_{T}(M)$ and hence defines an morphism on cohomology

$$
\tau^{\#} f^{\#}: H_{T}^{*}(N) \rightarrow H_{T}^{*}(M)
$$

If the map $f: M \rightarrow N$ is a diffeomorphism, then $\tau^{\#} f^{\#}$ is an isomorphism.

Another property of $f^{\#}$ which we will need if the following. If $T_{e}$ is a closed subgroup of $T$, with Lie algebra $\mathfrak{t}_{e}$, then one has restriction maps

$$
\Omega_{T} \rightarrow \Omega_{T_{e}},\left.\quad p \rightarrow p\right|_{t_{e}}
$$

and these induce maps in cohomology. If $\left.\tau\right|_{T_{e}}$ is the identity, then the diagram

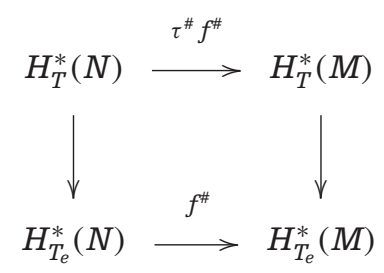

commutes.

We will apply these observations to the fibers of the $T$-equivariant fiber bundle $M \rightarrow B$, and we begin by recalling a few elementary facts about holonomy. By equipping $M$ with a $T$-invariant Riemannian metric, we obtain for each $m \in M$ an orthonormal complement in $T_{m} M$ of the tangent space at $m$ to the fiber of $\pi$, that is, an "Ehresman connection". Thus, if $p$ and $q$ are points of $B$ and $\gamma$ is a curve joining $p$ to $q$ we obtain, by parallel transport, a diffeomorphism $f_{\gamma}: F_{p} \rightarrow F_{q}$, where $f_{\gamma}(m)$ is the terminal point of the horizontal curve in $M$ projecting onto $\gamma$ and having $m$ as its initial point. Moreover, if $\gamma$ and $\gamma^{\prime}$ are homotopic curves joining $p$ to $q$, then the diffeomorphisms $f_{\gamma}$ and $f_{\gamma^{\prime}}$ are isotopic, that is, the isotopy class of $f_{\gamma}$ depends only on the homotopy class of $\gamma$.

Suppose now that the base $B$ is GKM, $p$ and $q$ are adjacent vertices of $\Gamma_{B}, e$ is the edge joining them and $S$ the two-sphere corresponding to this edge. We can then 
choose $\gamma$ to be a longitudinal line on $S$ joining the South pole $p$ to the North pole $q$; since this line is unique up to homotopy, we obtain an intrinsically defined isotopy class of diffeomorphisms of $F_{p}$ onto $F_{q}$. Moreover, since the Ehresman connection on $M$ is $T$-invariant and $T_{e}$ fixes $\gamma$, the maps in this isotopy class are $T_{e}$-invariant.

Definition 4.1. Let $M \rightarrow B$ be a $T$-equivariant fiber bundle over a GKM space $B$. Let $e=(p, q)$ be an edge of the GKM graph $\Gamma_{B}$. The $T$-equivariant fiber bundle $M \rightarrow B$ is balanced at $e$ if there exists a diffeomorphism $f_{e}$ in the isotopy class defined above and an automorphism $\tau_{e}$ of $T$ such that $f_{e}$ intertwines the $T$-action on $F_{p}$ with the $\tau_{e}$-twisted action of $T$ on $F_{q}$ :

$$
f_{e}(a x)=\tau_{e}(a) f_{e}(x)
$$

for all $a \in \tau_{e}$ and $x \in F_{p}$. The fiber bundle $M \rightarrow B$ is balanced if it is balanced at all edges of $\Gamma_{B}$.

It is clear that this $\tau_{e}$, if it exists, is unique and restricts to the identity on $T_{e}$.

Suppose now that the $T$-equivariant bundle $M \rightarrow B$ is balanced. Let $\operatorname{Aut}\left(F_{p}\right)$ be the group of isotopy classes of diffeomorphisms of $F_{p}$, Aut(T) the group of automorphisms of $T$, and $\pi_{1}(\Gamma, p)$ the group of paths $\gamma=\left(e_{1}, e_{2}, \ldots, e_{k}\right)$ of oriented edges in $\Gamma$ starting and ending at $p$. Given a path $\gamma$ in $\pi_{1}(\Gamma, p)$ we have for each $j$ a ring isomorphism

$$
\tau_{e_{j}}^{\#} f_{e_{j}}^{\#}: H_{T}^{*}\left(F_{p_{j+1}}\right) \rightarrow H_{T}^{*}\left(F_{p_{j}}\right)
$$

$p_{j}$ and $p_{j+1}$ being the initial and terminal vertices of $e_{j}$, and by composing these maps we obtain a ring automorphism, $\tau_{e_{k}}^{\#} f_{e_{k}}^{\#} \circ \cdots \circ \tau_{e_{1}}^{\#} f_{e_{1}}^{\#}$, of $H_{T}^{*}\left(F_{p}\right)$. Moreover, by (4.4) we can rewrite the factors in this product as $\tau_{\gamma}^{\#} f_{\gamma}^{\#}$ where $\tau_{\gamma}=\tau_{e_{1}} \circ \cdots \circ \tau_{e_{k}}$ and $f_{\gamma}=f_{e_{1}} \circ \cdots \circ f_{e_{k}}$. Thus the map $\gamma \rightarrow \tau_{\gamma}^{\#} f_{\gamma}^{\#}$ gives one a holonomy action of $\pi_{1}(\Gamma, p)$ on $H_{T}^{*}\left(F_{p}\right)$. We will denote the image of this map by $W_{p}$ and call it the Weyl group of $p$. As a subgroup of $\operatorname{Aut}\left(F_{p}\right) \times \operatorname{Aut}(T), W_{p}$ acts on $H_{T}^{*}\left(F_{p}\right)$ and on $\mathbb{S}\left(\mathfrak{t}^{*}\right)$. The main result of this section is the following theorem.

Theorem 4.2. Let $\pi: M \rightarrow B$ be a balanced $T$-equivariant fiber bundle, such that the base $B$ is a simply connected GKM space and the total space $M$ is equivariantly formal. Moreover, suppose that the fiber $F_{p}$ over a fixed point $p \in B^{T}$ is also equivariantly formal as a $T$-space. Then

$$
H_{T}^{*}(M) \simeq H_{T}^{*}\left(F_{p}\right)^{W_{p}} \otimes_{\mathbb{S}\left(t^{*}\right)^{W p}} H_{\alpha}\left(\Gamma_{B}\right)
$$


Proof. By (1.7), $H_{T}^{*}(M)$ is isomorphic to the subring of

$$
\operatorname{Maps}_{0}=\left\{\phi: B^{T} \rightarrow \bigoplus_{q \in B^{T}} H_{T}^{*}\left(F_{q}\right) \mid \phi(q) \in H_{T}^{*}\left(F_{q}\right) \text { for all } q\right\}
$$

given by the conditions of Theorem 1.3. We identify $H_{T}^{*}(M)$ with that subring and consider $H_{T}^{*}(M) \subset \operatorname{Maps}_{0}$.

Let $c_{p} \in H_{T}^{*}\left(F_{p}\right)^{W_{p}}$. For $q \in B^{T}$, let $\gamma_{p, q}$ be a path in $\Gamma$ joining $p$ to $q$, and let $c_{q} \in H_{T}^{*}\left(F_{q}\right)$ be the class obtained by transporting $c_{p}$ along $\gamma_{p, q}$. Because $c_{p}$ is $W_{p^{-}}$ invariant, this class $C_{q}$ is independent of the chosen path $\gamma_{p, q}$. Consider

$$
\tilde{C}: B^{T} \rightarrow \bigoplus_{q \in B^{T}} H_{T}^{*}\left(F_{q}\right), \quad \tilde{C}(q)=c_{q}
$$

If $q_{1}$ and $q_{2}$ are adjacent vertices in $\Gamma_{B}$ and $e$ is the edge joining $q_{1}$ to $q_{2}$, then $c_{q_{1}}=\tau_{e}^{*} f_{e}^{*} c_{q_{2}}$ and hence by the commutative diagram (4.7), the images of $c_{q_{1}}$ and $c_{q_{2}}$ in the quotient space

$$
H_{T}^{*}\left(F_{q_{1}}\right) /\left\langle\alpha_{e}\right\rangle=H_{T}^{*}\left(F_{q_{2}}\right) /\left\langle\alpha_{e}\right\rangle
$$

are the same. Using Theorem 1.3, we conclude that $\tilde{c} \in H_{T}^{*}(M)$. We have therefore defined an injective morphism of rings

$$
H_{T}^{*}\left(F_{p}\right)^{W_{p}} \rightarrow H_{T}^{*}(M)
$$

and this map is also a morphism of $\mathbb{S}\left(\mathfrak{t}^{*}\right)^{W_{p}}$-modules. Tensoring with the pull-back

$$
H_{\alpha}\left(\Gamma_{B}\right) \simeq H_{T}^{*}(B) \stackrel{\pi^{*}}{\rightarrow} H_{T}^{*}(M)
$$

we obtain a morphism of rings

$$
\Phi: H_{T}^{*}\left(F_{p}\right)^{W_{p}} \otimes_{\mathbb{S}\left(t^{*}\right)^{W_{p}}} H_{\alpha}\left(\Gamma_{B}\right) \rightarrow H_{T}^{*}(M)
$$

which acts on decomposable elements $c_{p} \otimes \omega$ by

$$
\Phi\left(c_{p} \otimes \omega\right)(q)=\omega(q) c_{q}
$$

We will show that $\Phi$ is an isomorphism. 
We first show that $\Phi$ is injective. Consider the double complex $\left(\Omega_{T}\left(F_{p}\right), d, \delta\right)$, with $(d h)(\xi)=d(h(\xi))$ and $(\delta h)(\xi)=\iota_{\xi^{\#}} h(\xi)$. Let $\gamma \in \pi_{1}(\Gamma, p)$ be a loop in $\Gamma$ starting and ending at $p$ and $f_{\gamma}: F_{p} \rightarrow F_{p}$ and $\tau_{\gamma}: T \rightarrow T$ the maps obtained by composing the corresponding transition maps along the edges of $\gamma$. The action of $\gamma$ on $\Omega_{T}\left(F_{p}\right)$, given by

$$
(\gamma \cdot h)(\xi)=f_{\gamma}^{*} h\left(\left(\tau_{\gamma}\right)_{*}(\xi)\right)
$$

preserves the bigrading of the double complex and commutes with the coboundary operators $d$ and $\delta$. Since $F_{p}$ is by assumption equivariantly formal,

$$
H_{T}^{*}\left(F_{p}\right) \simeq H^{*}\left(F_{p}\right) \otimes \mathbb{S}\left(\mathfrak{t}^{*}\right)
$$

as $\mathbb{S}\left(\mathfrak{t}^{*}\right)$-modules. Moreover, since the $W_{p}$-action on $\Omega_{T}\left(F_{p}\right)$ commutes with the coboundary operators $d$ and $\delta$, the isomorphism (4.9) is $W_{p}$-equivariant. Since $B$ is simply connected, the diffeomorphisms $f_{\gamma}: F_{p} \rightarrow F_{p}$ are (nonequivariantly) isotopic to the identity, so they act trivially on $H^{*}\left(F_{p}\right)$, and therefore

$$
H_{T}^{*}\left(F_{p}\right)^{W_{p}} \simeq H^{*}\left(F_{p}\right) \otimes \mathbb{S}\left(\mathfrak{t}^{*}\right)^{W_{p}}
$$

Hence a basis $\tilde{C}_{1, p}, \ldots, \tilde{C}_{N, p}$ of $H^{*}\left(F_{p}\right)$ generates a basis $C_{1, p}, \ldots, C_{N, p}$ of $H_{T}^{*}\left(F_{p}\right)^{W_{p}}$ as a free module over $\mathbb{S}\left(\mathfrak{t}^{*}\right)^{W_{p}}$.

Every element $\eta \in H_{T}^{*}\left(F_{p}\right)^{W_{p}} \otimes_{\mathbb{S}\left(t^{*}\right)^{W_{p}}} H_{\alpha}\left(\Gamma_{B}\right)$ is of the form

$$
\eta=\sum_{k=1}^{N} c_{k, p} \otimes \omega_{k}
$$

for some classes $\omega_{k} \in H_{\alpha}\left(\Gamma_{B}\right)$. If $\eta \in \operatorname{ker} \Phi$, then for every $q \in B^{T}$,

$$
\sum_{k=1}^{N} \omega_{k}(q) c_{k, q}=\Phi(\eta)(q)=0
$$

But for every $q$ the classes $\left\{c_{1, q}, \ldots, c_{N, q}\right\}$ are a free set of generators for the $\mathbb{S}\left(\mathfrak{t}^{*}\right)$-module $H_{T}^{*}\left(F_{q}\right)$, and that implies that $\omega_{1}=\cdots=\omega_{N}=0$, hence $\eta=0$. This proves that $\Phi$ is injective. 
To prove the surjectivity, we keep track of bi-degrees. Consider the composition

$$
\Psi: H^{*}\left(F_{p}\right) \otimes H_{T}^{*}(B) \rightarrow H_{T}^{*}\left(F_{p}\right)^{W_{p}} \otimes_{\mathbb{S}\left(\mathfrak{t}^{*}\right)^{W_{p}}} H_{\alpha}\left(\Gamma_{B}\right) \stackrel{\Phi}{\rightarrow} H_{T}^{*}(M)
$$

the first arrow is the linear map that sends $\tilde{c}_{j, p} \otimes \omega$ to $c_{j, p} \otimes \omega$ for all $j=1, \ldots, N$. Therefore, $\Psi\left(\tilde{c}_{j, p} \otimes \omega\right)(q)=c_{j, p}(q) \omega(q)$ for all $q \in V_{\Gamma}$, for all $j=1, \ldots, N$.

By assumption, the Serre-Leray spectral sequence associated with the fiber bundle $\pi$ collapses at its $E_{2}$ stage. The $E_{2}$ term of this sequence is $H^{*}\left(F_{p}\right) \otimes H_{T}^{*}(B)$ and the $E_{\infty}$ term is $H_{T}^{*}(M)$, hence

$$
\bigoplus_{j+k=i} H^{j}\left(F_{p}\right) \otimes H_{\alpha}^{k}\left(\Gamma_{B}\right)=H_{T}^{i}(M) .
$$

Since $\Psi$ preserves the grading and on each homogeneous component is an injective linear map between spaces of the same dimension, we conclude that $\Psi$ is surjective, and therefore $\Phi$ is surjective as well.

Apropos of the assumption that the $F_{p}$ 's are equivariantly formal as $T$ spaces, we note:

(i) Since the fiber bundle, $M \rightarrow B$, is balanced, it suffices to assume this just for the "base" fiber, $F_{p_{0}}$, above a single $p_{0} \in B^{T}$.

(ii) If $M$ is a Hamiltonian $T$-space, then the fibers over points in $B^{T}$ are Hamiltonian $T$-spaces as well, hence are equivariantly formal. (Note that in particular if $M$ is a Hamiltonian GKM space, then the fibers over points in $B^{T}$ are Hamiltonian GKM spaces.)

\section{Homogeneous Fiber Bundles}

We apply the results of the previous section to homogeneous fiber bundles, and we start by recalling some of the notation and results of Guillemin et al. [4].

Let $G$ be a compact connected semisimple Lie group, $T$ its Cartan subgroup, and $K$ a closed subgroup of $G$ containing $T$. Then, as asserted above, $G / K$ is a GKM space. The proof of this consists essentially of describing explicitly the GKM structure of $G / K$ in terms of the Weyl groups of $G$ and $K$. We first note that for $K=T$, that is, for the generalized flag variety $M=G / T$, the fixed point set, $M^{T}$, is just the orbit of $N(T)$, the normalizer of $T$ in $G$, through the identity coset, $p_{0}$, and hence $M^{T}$ can be 
identified with $N(T) / T=W_{G}$. Let $\Delta_{G}$ be the set of roots, and $\Delta_{G}^{+}$a choice of positive roots. To show that $M$ is $G K M$ it suffices to check the GKM condition $p_{0}$. To do so, we identify the tangent space $T_{p_{0}} M$ with $\mathfrak{g} / \mathfrak{t}$ and identify $\mathfrak{g} / \mathfrak{t}$ with the sum of the positive root spaces

$$
\mathfrak{g} / \mathfrak{t}=\bigoplus_{\alpha \in \Delta_{G}^{+}} \mathfrak{g}_{\alpha},
$$

the $\alpha^{\prime} \mathrm{s}$ being the weights of the isotropy representation of $T$ on $\mathfrak{g} / \mathfrak{t}$. It then follows from a standard theorem in Lie theory that the weights are pairwise independent and this in turn implies that condition $\left(\mathrm{c}^{\prime}\right)$ is satisfied at $p_{0}$.

To see what the edges of the GKM graph are at $p_{0}$ let $\chi_{\alpha}: T \rightarrow S^{1}$ be the character homomorphism

$$
\chi_{\alpha}(t)=\operatorname{exp~i} \alpha(t)
$$

let $H_{\alpha}$ be its kernel, and $G_{\alpha}$ the semisimple component of the centralizer $C\left(H_{\alpha}\right)$ of $H_{\alpha}$ in $G$. Then $G_{\alpha}$ is either $\mathrm{SU}(2)$ or $\mathrm{SO}(3)$, and in either case $G_{\alpha} p_{0} \simeq \mathbb{C} P^{1}$. However since $G_{\alpha}$ centralizes $H_{\alpha}, G_{\alpha} p_{0}$ is $H_{\alpha}$-fixed and hence is the connected component of $M^{H_{\alpha}}$ containing $p_{0}$. Thus, the oriented edges of the GKM graph of $M$ with initial point $p_{0}$ can be identified with the elements of $\Delta_{G}^{+}$and the axial function becomes the function which labels by $\alpha$ the oriented edge $G_{\alpha} p_{0}$. Moreover, under the identification $M^{T}=W_{G}$, the vertices that are joined to $p_{0}$ by these edges are of the form $\sigma_{\alpha} p_{0}$, where $\sigma_{\alpha} \in W_{G}$ is the reflection which leaves fixed the hyperplane ker $\alpha \subseteq \mathfrak{t}$ and maps $\alpha$ to $-\alpha$.

Letting $a \in N(T)$ and letting $p=a p_{0}$ one obtains essentially the same description of the GKM graph at $p$. Namely, denoting this graph by $\Gamma$, the following are true:

(1) the maps, $a \in N(T) \rightarrow a p_{0}$ and $a \in N(T) \rightarrow w \in N(T) / T$, set up a 1-to-1 correspondence between the vertices, $M^{T}$, of $\Gamma$ and the elements of $W_{G}$;

(2) two vertices, $w$ and $w^{\prime}$, are on a common edge if and only if $w^{\prime}=w \sigma_{\alpha}$ for some $\alpha \in \Delta_{G}^{+} ;$

(3) the edges of $\Gamma$ containing $p=a p_{0}$ are in one-one correspondence with elements of $\Delta_{G}^{+}$;

(4) for $\alpha \in \Delta_{G}^{+}$the stabilizer group of the edge corresponding to $\alpha$ is $a H_{\alpha} a^{-1}$.

Via the fiber bundle $G / T \rightarrow G / K$ one obtains essentially the same picture for $G / K$. Namely let $\Delta_{G, K}^{+}=\Delta_{G}^{+} \backslash \Delta_{K}^{+}$. 
Theorem 5.1 ([4, Theorem 2.4]). The homogeneous space $G / K$ is a GKM space with GKM graph $\Gamma$, where

(1) the vertices of $\Gamma$ are in 1-to-1 correspondence with the elements of $W_{G} / W_{K}$;

(2) two vertices $[w]$ and $\left[w^{\prime}\right]$ are on a common edge if and only if $\left[w^{\prime}\right]=\left[w \sigma_{\alpha}\right]$ for some $\alpha \in \Delta_{G, K}^{+}$;

(3) the edges of $\Gamma$ containing the vertex $[w]$ are in 1-to-1 correspondence with the roots in $\Delta_{G, K}^{+}$;

(4) if $\alpha$ is such a root the the stabilizer group of the $\mathbb{C} P^{1}$ corresponding to the edge is $a H_{\alpha} a^{-1}$ where $a$ is a preimage in $N(T)$ of $w \in W_{G}$.

Remark 5.2. The GKM graph that we have just described is not simple in general, that is, it will in general have more than one edge joining two adjacent vertices. There is, however, a simple sufficient condition for simplicity.

Theorem 5.3 ([4]). If $K$ is a stabilizer group of an element of $\mathfrak{t}^{*}$, that is, if $G / K$ is a coadjoint orbit, then the graph we have constructed above is simple.

Proposition 5.4. Let $K_{1}$ be a closed subgroup of $K$. Then the fiber bundle

$$
G / K_{1} \rightarrow G / K
$$

is balanced.

Proof. To show that this is balanced it suffices to show that it is balanced at the edges going out of the identity coset, $p_{0}$. However, if $e$ is the edge joining $p_{0}$ to $\sigma_{\alpha} p_{0}$ and $a$ is the preimage of $\sigma_{\alpha}$ in $N(T)$ then conjugation by a maps the fiber, $F_{p_{0}}=K / K_{0}$ of (5.2) at $p_{0}$ onto the fiber $F_{p}:=a K / a K_{0}$ of (5.2) at $p=\sigma_{\alpha} p_{0}$, and conjugates the action of $\Gamma$ on $F_{p_{0}}$ to the twisted action, $a T a^{-1}$, of $T$ on $F_{p}$. Moreover, since $a$ is in the centralizer of $H_{\alpha}$, this twisted action, restricted to $H_{\alpha}$, coincides with the given action of $H_{\alpha}$, that is, if $T_{e}=H_{\alpha}$, conjugation by $a$ is a $T_{e}$-equivariant isomorphism of $F_{p_{0}}$ onto $F_{p}$. Hence the fiber bundle (5.2) is balanced.

For the homogeneous fiber bundles described in this section, the assumption that the fibers $F_{p}$ are equivariantly formal as $T$-spaces is equivalent to the assumption that the $F_{p}$ 's are GKM. To see this, we note that if $G / K$ is equivariantly formal then $(G / K)^{T}$ has to be nonempty by Guillemin and Sternberg [7, Theorem 11.4.5] and hence 
for some $g \in G, g^{-1} \mathrm{Tg} \subseteq K$. In other words $K$ is of maximal rank and hence by the theorem in [4] that we cited above $G / K$ is GKM.

\section{Examples}

The results of this paper are closely related to the combinatorial results of our recent article [5]. More explicitly, in [5] we develop a GKM theory for fiber bundles in which the objects involved: the base, the fiber and the total space of the fiber bundle, are GKM graphs. We then formulate, in this context, a combinatorial notion of "balanced", show that one has an analog of the isomorphism (1.11) and use this fact to define some new combinatorial invariants for the classical flag varieties of type $A_{n}, B_{n}, C_{n}$, and $D_{n}$. In this section, we will give a brief account of how these invariants can be defined geometrically by means of the techniques developed above.

Example 1. Let $G=\mathrm{SU}(n+1), K=T$, the Cartan subgroup of diagonal matrices in $\mathrm{SU}(n+1)$, and $K_{1}=S\left(S^{1} \times U(n)\right)$. Then $G / K_{1} \simeq \mathbb{C} P^{n}$, the complex projective space. The Weyl group of $\mathrm{SU}(n+1)$ is isomorphic to $S_{n+1}$, the group of permutations on $n+1$ elements. Let $\mathcal{A}_{n}=G / K$ be the generic coadjoint orbit of type $A_{n}$; then $\mathcal{A}_{n} \simeq \mathcal{F l}\left(\mathbb{C}^{n+1}\right)$, the variety of complete flags in $\mathbb{C}^{n+1}$. The fiber bundle

$$
\pi: \mathcal{A}_{n} \simeq G / K \rightarrow G / K_{1} \simeq \mathbb{C} P^{n}
$$

sends a flag $V_{\bullet}$ to its one-dimensional subspace $V_{1}$. The fiber over a line $L$ in $\mathbb{C} P^{n}$ is $\mathcal{F} l\left(\mathbb{C}^{n+1} / L\right) \simeq \mathcal{F} l\left(\mathbb{C}^{n}\right) \simeq \mathrm{SU}(n) / T^{\prime}$, where $T^{\prime}$ is the Cartan subgroup of diagonal matrices in $\mathrm{SU}(n)$. The fibers inherit a $T$-action from $\mathcal{F l}\left(\mathbb{C}^{n+1}\right)$, but are not $T$-equivariantly isomorphic. If $p$ and $q$ are fixed points for the $T$-action on $\mathbb{C} P^{n}$, then the fibers $F_{p}$ and $F_{q}$ are $T_{e}$-equivariantly isomorphic, where $T_{e}$ is the subtorus fixing the $\mathbb{C} P^{1}$ in $\mathbb{C} P^{n}$ with poles $p$ and $q$. The Weyl group $W_{p}$ of the fiber at $p$ is isomorphic to $S_{n}$, the Weyl group of SU(n), and the holonomy action of $W_{p}$ on the equivariant cohomology of the fiber is equivalent to the induced action of $S_{n}$ on the equivariant cohomology of the flag variety $\mathcal{F} l\left(\mathbb{C}^{n}\right)$.

We can iterate this fiber bundle and construct a tower of fiber bundles

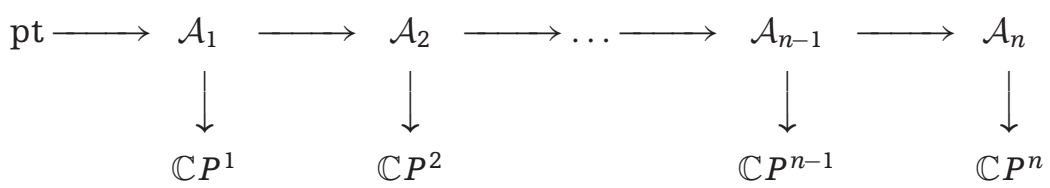


Using this tower, we construct a basis of invariant classes on $\mathcal{A}_{n}$ by repeatedly applying the isomorphism (1.10). A typical stage in the process is the following. By (1.10), we have

$$
H_{T}\left(\mathcal{A}_{k}\right)=H_{T}\left(\mathcal{A}_{k-1}\right)^{S_{k}} \otimes_{\mathbb{S}\left(\mathbf{t}^{*}\right)^{S_{k}}} H_{T}\left(\mathbb{C} P^{k}\right)
$$

Suppose we have constructed a basis of invariant classes on $\mathcal{A}_{k-1}$; this is trivial for $\mathcal{A}_{0}=$ pt. We use, as a basis for $H_{T}\left(\mathbb{C} P^{k}\right)$, classes represented by powers of the equivariant symplectic form $\omega-\tau \in \Omega_{T}^{2}\left(\mathbb{C} P^{k}\right)$. The pull-backs of these classes to $\mathcal{A}_{k}$ are invariant under the holonomy action, and the classes given by the isomorphism (1.10) form a basis of the equivariant cohomology of $\mathcal{A}_{k}$. As shown in [5], this basis consists of classes that are invariant under the corresponding holonomy action. By iterating this process, we obtain an $\mathbb{S}\left(\mathfrak{t}^{*}\right)$-basis of $H_{T}\left(\mathcal{A}_{n}\right)$ consisting of $S_{n+1}$-invariant classes. The combinatorial version of this construction is given in [5, Section 5.1].

Example 2. Let $G=\mathrm{SO}(2 n+1), K=T$ a maximal torus in $G$, and $K_{1}=\mathrm{SO}(2) \times \mathrm{SO}(2 n-1)$. Then $G / K_{1} \simeq \mathrm{Gr}_{2}^{+}\left(\mathbb{R}^{2 n+1}\right)$, the Grassmannian of oriented two planes in $\mathbb{R}^{2 n+1}$. Let $W_{n}$ be the Weyl group of $\mathrm{SO}(2 n+1)$, that is, the group of signed permutations on $n$ elements. Let $\mathcal{B}_{n}=G / K$ be the generic coadjoint orbit of type $B_{n}$ and

$$
\pi: \mathcal{B}_{n} \simeq G / K \rightarrow G / K_{1} \simeq \mathrm{Gr}_{2}^{+}\left(\mathbb{R}^{2 n+1}\right)
$$

the natural projection. Note that the fibers are isomorphic to $\mathcal{B}_{n-1}$, but not isomorphic as $T$-spaces, since the $T$-action on the pre-image of the $T$-fixed points of $\mathrm{Gr}_{2}^{+}\left(\mathbb{R}^{2 n+1}\right)$ changes. Moreover, for every $T$-fixed point $p \in \mathrm{Gr}_{2}^{+}\left(\mathbb{R}^{2 n+1}\right)$, the Weyl group $W_{p}$ of the fiber $F_{p}$ is isomorphic to $W_{n-1}$. Just as in the previous example, we have the following tower of fiber bundles:

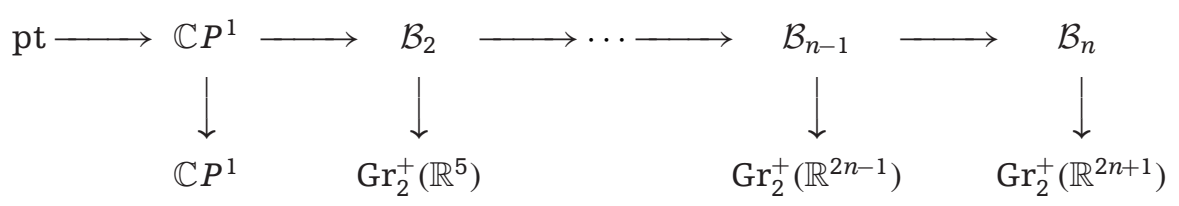

and we can use this tower to produce a basis of invariant classes on $\mathcal{B}_{n}$. Namely, by (1.10), we have

$$
H_{T}\left(\mathcal{B}_{k}\right)=H_{T}\left(\mathcal{B}_{k-1}\right)^{W_{k-1}} \otimes_{\mathbb{S}\left(t^{*}\right)^{W_{k-1}}} H_{T}\left(\mathrm{Gr}_{2}^{+}\left(\mathbb{R}^{2 k+1}\right)\right)
$$

Since the classes represented by powers of the equivariant symplectic form $\omega-\tau \in \Omega_{T}^{2}\left(\mathrm{Gr}_{2}^{+}\left(\mathbb{R}^{2 k+1}\right)\right)$ are a $W_{k}$-invariant basis for $H_{T}\left(\mathrm{Gr}_{2}^{+}\left(\mathbb{R}^{2 k+1}\right)\right)$, their pull-backs are 
classes on $\mathcal{B}_{k}$ invariant under the holonomy action. Thus, we can repeat the same argument of the previous example and produce a basis for $H_{T}\left(\mathcal{B}_{n}\right)$ consisting of $W_{n}$-invariant classes.

Example 3. Let $G=\operatorname{Sp}(n)$ be the symplectic group, $K=T$ a maximal torus in $G$, and $K_{1}=S^{1} \times \operatorname{Sp}(n-1)$. Then $G / K_{1} \simeq \mathbb{C} P^{2 n-1}$. Let $W_{n}$ be the Weyl group of $\operatorname{Sp}(n)$, that is, the group of signed permutations on $n$ elements. Let $\mathcal{C}_{n}=G / K$ be the generic coadjoint orbit of type $C_{n}$ and

$$
\pi: \mathcal{C}_{n} \simeq G / K \rightarrow G / K_{1} \simeq \mathbb{C} P^{2 n-1}
$$

the natural projection. For every $T$-fixed point $p \in \mathbb{C} P^{2 n-1}$, the Weyl group acting on the fiber $F_{p}$ is isomorphic to $W_{n-1}$. Since the fibers are isomorphic to $\mathcal{C}_{n-1}$, we obtain the following tower of fiber bundles:

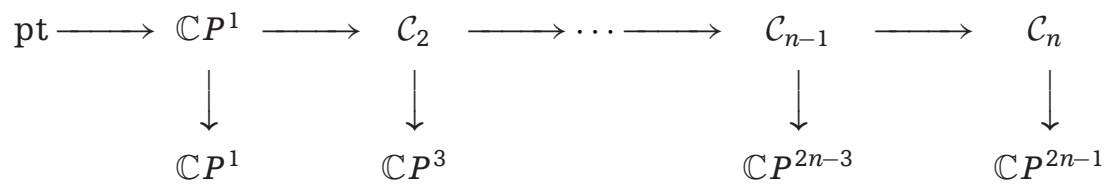

By (1.10), we have that

$$
H_{T}\left(\mathcal{C}_{k}\right)=H_{T}\left(\mathcal{C}_{k-1}\right)^{W_{k-1}} \otimes_{\mathbb{S}\left(\mathrm{t}^{*}\right)^{W_{k-1}}} H_{T}\left(\mathbb{C} P^{2 k-1}\right)
$$

By taking classes represented by powers of the equivariant symplectic form $\omega-\tau \in \Omega_{T}^{2}\left(\mathbb{C} P^{2 k-1}\right)$ we obtain a $W_{k}$-invariant basis of $H_{T}\left(\mathbb{C} P^{2 k-1}\right)$, and iterating the same procedure as before, a $W_{n}$-invariant basis of $H_{T}\left(\mathcal{C}_{n}\right)$.

Example 4. Let $G=\mathrm{SO}(2 n), K=T$ a maximal torus in $G$, and $K_{1}=\mathrm{SO}(2) \times \mathrm{SO}(2 n-2)$. Then $G / K_{1} \simeq \mathrm{Gr}_{2}^{+}\left(\mathbb{R}^{2 n}\right)$, the Grassmannian of oriented two planes in $\mathbb{R}^{2 n}$. Let $W_{n}$ be the Weyl group of $\mathrm{SO}(2 n)$, that is, the subgroup of the signed permutation group of $n$ elements, consisting of an even number of sign changes. Let $\mathcal{D}_{n}=G / K$ be the generic coadjoint orbit of type $D_{n}$ and

$$
\pi: \mathcal{D}_{n} \simeq G / K \rightarrow G / K_{1} \simeq \mathrm{Gr}_{2}^{+}\left(\mathbb{R}^{2 n}\right)
$$

the natural projection. For every $T$-fixed point $p \in \mathrm{Gr}_{2}^{+}\left(\mathbb{R}^{2 n}\right)$, the Weyl group acting on the fiber $F_{p}$ is isomorphic to $W_{n-1}$. Since the fibers are isomorphic to $\mathcal{D}_{n-1}$, we obtain the 
following tower of fiber bundles:

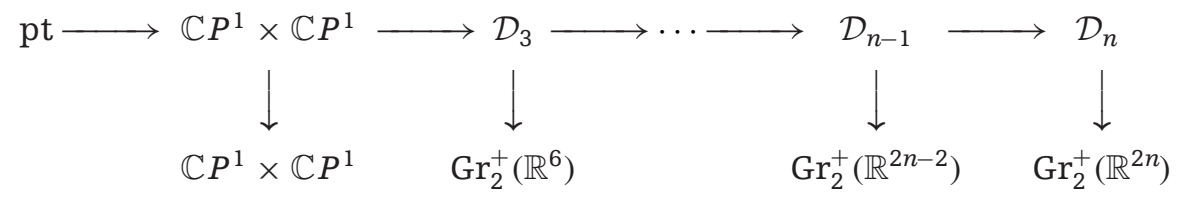

By (1.10), we have that

$$
H_{T}\left(\mathcal{D}_{k}\right)=H_{T}\left(\mathcal{D}_{k-1}\right)^{W_{k-1}} \otimes_{\mathbb{S}\left(\mathfrak{t}^{*}\right)^{W_{k-1}}} H_{T}\left(\mathrm{Gr}_{2}^{+}\left(\mathbb{R}^{2 k}\right)\right)
$$

By repeating an argument similar to the other examples, in order to produce a $W_{n}$-invariant basis of $H_{T}\left(\mathcal{D}_{n}\right)$, it is sufficient to exhibit a $W_{k}$-invariant basis of $H_{T}\left(\mathrm{Gr}_{2}^{+}\left(\mathbb{R}^{2 k}\right)\right)$ for every $k \geqslant 2$. To do this, let $x_{1}-x_{2}, \ldots, x_{k-1}-x_{k}, x_{k-1}+x_{k}$ be a choice of simple roots of type $D_{k}$. Then the classes represented by powers of the equivariant symplectic form $\omega-\tau \in \Omega_{T}^{2}\left(\mathrm{Gr}_{2}^{+}\left(\mathbb{R}^{2 n}\right)\right)$ together with the class $\eta \in H_{T}^{2 n-2}\left(\mathrm{Gr}_{2}^{+}\left(\mathbb{R}^{2 n}\right)\right)$ whose restriction at the fixed points is given by $x_{1} \cdots x_{k} \tau^{-1}$, form a $W_{k}$-invariant basis of $H_{T}\left(\mathrm{Gr}_{2}^{+}\left(\mathbb{R}^{2 n}\right)\right)$.

In [5], we also show how these iterated invariant classes relate to a better known family of classes generating the equivariant cohomology of flag varieties, namely the equivariant Schubert classes.

\section{References}

[1] Atiyah, M. F. and I. G. Macdonald. Introduction to Commutative Algebra. Reading, MA: Addison-Wesley, 1969.

[2] Chang, T. and T. Skjelbred. "The topological Schur lemma and related results." Annals of Mathematics 100, no. 2 (1974): 307-21.

[3] Goresky, M., R. Kottwitz, and R. MacPherson. "Equivariant cohomology, Koszul duality, and the localization theorem." Inventiones Mathematicae 131, no. 1 (1998): 25-83.

[4] Guillemin, V., T. Holm, and C. Zara. "A GKM description of the equivariant cohomology ring of a homogeneous space." Journal of Algebraic Combinatorics 23, no. 1 (2006): $21-41$.

[5] Guillemin, V., S. Sabatini, and C. Zara. "Cohomology of GKM fiber bundles." Journal of Algebraic Combinatorics 35, no. 1 (2012): 19-59.

[6] Guillemin, V., S. Sabatini, and C. Zara. "Equivariant $K$-theory of GKM bundles." Annals of Global Analysis and Geometry. DOI:10.1007/s10455-012-9331-3. arXiv:1203.1027v1 [math.KT]. 
3910 V. Guillemin et al.

[7] Guillemin, V. and S. Sternberg. Supersymmetry and Equivariant de Rham Theory. Mathematics Past and Present. Berlin: Springer, 1999.

[8] Guillemin, V. and C. Zara. “G-actions on graphs." International Mathematics Research Notes no. 10 (2001): 519-42.

[9] Sabatini, S. and S. Tolman. "New techniques for obtaining Schubert-type formulas for Hamiltonian manifolds." (2010): preprint arXiv:1004.4543v1 [math.SG]. 